\title{
First steps toward the development of SONATE, a Compact Accelerator driven Neutron Source
}

\author{
Lö̈c Thulliez ${ }^{1, *}$, Alain Letourneau ${ }^{1}$, Jérôme Schwindling ${ }^{1}$, Nicolas Chauvin ${ }^{1}$, Nadia Sellami ${ }^{1}$, Frédéric Ott $^{2}$, Alain \\ Menelle $^{2}$, and Burkhard Annighöfer ${ }^{2}$ \\ ${ }^{1}$ IRFU, CEA, Université Paris-Saclay, 91191 Gif-sur-Yvette, France \\ ${ }^{2}$ LLB, CEA, CNRS, Université Paris-Saclay, 91191 Gif-sur-Yvette, France
}

\begin{abstract}
.
Facilities providing bright thermal neutron beams are of primary importance for various research topics such as condensed matter experiments, neutron-imaging or medical applications. Currently these are mainly spallation sources and nuclear reactors. However, these later facilities are ageing and the political context does not favor the building of new ones. This is the case in CEA-Saclay (France), where the Orphee reactor is planned to shutdown in 2019. Therefore, another local facility, affordable by one country, able to provide high brilliance neutron beams has to be built. At CEA-Saclay, a compact accelerator driven neutron source, SONATE, is investigated in taking advantage of the IPHI accelerator able to deliver a $3 \mathrm{MeV}$ proton beam with an intensity up to $100 \mathrm{~mA}$. In the future, SONATE is foreseen to operate with $20 \mathrm{MeV}$ protons to increase the neutron brightness. In addition to the difficulties to operate such high intensity accelerators, the other challenges regard the target-moderator-reflector (TMR) design which is crucial to maximize the neutron flux at the detector location. At CEA-Saclay, several experiments were performed between 2016 and 2019 with the IPHI accelerator. Geant 4 simulations were also developed. They demonstrate the feasibility of such concept and enable to find the best TMR configuration for the future SONATE facility. These developments are reported in this article.
\end{abstract}

\section{Introduction}

In Europe, after the ILL nuclear reactor shutdown foreseen in 2030-2040 (optimistic scenario from [1]), the neutron beam time will drop roughly by $40 \%$. The neutron beam time in Europe will then depend mainly on four big facilities: ESS (Sweden, start foreseen in 2024), ISIS (UK), SINQ (Switzerland) and FRM-II (Germany). The beam time will then be expensive and dedicated to high impact experiments. In this context, the development of new small facilities affordable by one country are necessary to support the large neutron community. It is of primary importance to develop new experimental techniques and to prepare scientists to use the big facilities. Fission, spallation and fusion reaction based facilities could be a solution but are not suited as local facilities because of either political choices, their high costs or their low neutron yields. Compact accelerator driven neutron sources (CANS), based on (p/d,n) reaction with light nucleus targets, can now be competitive with nuclear reactors in term of neutron brightness because of the recent developments of high intensity accelerators, and at low cost (less than 200 Meuros).

In France at CEA-Saclay, since the Orphée reactor will shutdown in autumn 2019, a CANS is expected to be developed taking advantage of the IPHI proton accelerator. This installation is able to deliver protons with an energy

*e-mail: loic.thulliez@cea.fr
$\left(\mathrm{E}_{p}\right)$ of $3 \mathrm{MeV}$ and a high intensity up to $100 \mathrm{~mA}$, in continuous or pulsed mode. For this purpose a targetmoderator-reflector (TMR) assembly providing the highest well collimated thermal $\left(\mathrm{E}_{n}<100 \mathrm{meV}\right)$ neutron flux is searched for.

This paper starts by presenting the criteria leading to select the best suited target to generate the primary neutrons. Then the validation of the Geant4 simulation (version 10.04.p02) [2] is presented for different TMR configurations. Finally, the first optimization steps to find the best TMR configuration, for $3 \mathrm{MeV}$ proton beam of few $\mathrm{mA}$, are detailed focusing on the moderator and reflector materials and geometries.

\section{Target design criteria}

The target has to maximize the neutron yield $\left(\mathrm{Y}_{n}\right)$ for $3 \mathrm{MeV}$ protons and to handle the heat load deposited by the beam. At $\mathrm{E}_{p}=3 \mathrm{MeV}$, to maximize $\mathrm{Y}_{n}$ the target nucleus should have a low Coulomb barrier (a low nuclear charge) and a low reaction energy threshold (a low nucleon binding energy). To that respect the stable nuclei having the higher $(\mathrm{p}, \mathrm{n})$ reaction cross-section at $3 \mathrm{MeV}$ are ${ }^{7} \mathrm{Li}$ and ${ }^{9} \mathrm{Be}[4]$. Table 1 shows that for ${ }^{7} \mathrm{Li}$ and ${ }^{9} \mathrm{Be}$ thick target (thick enough to stop the protons inside the target), $\mathrm{Y}_{n}$ are respectively $2.38 \times 10^{-4} \mathrm{n} / \mathrm{p}$ and $5.23 \times 10^{-5}$ $\mathrm{n} / \mathrm{p}$ [3]. In increasing $\mathrm{E}_{p}$ to $5 \mathrm{MeV}, \mathrm{Y}_{n}$ will increase by a factor 4.2 and 9.5 respectively for ${ }^{7} \mathrm{Li}$ and ${ }^{9} \mathrm{Be}$, which 
Table 1: Neutronic and thermal properties for different material foreseen as a target. The neutron yields are from [3].

\begin{tabular}{cc|ccc|cc}
\hline \multicolumn{2}{c}{ General } & \multicolumn{2}{c}{ Neutronic } & \multicolumn{2}{c}{ Thermal } \\
\hline Nucleus & $\rho\left[\mathrm{g} / \mathrm{cm}^{3}\right]$ & $\mathrm{E}_{t h}[\mathrm{MeV}]$ & $\mathrm{Y}_{n}[\mathrm{n} / \mathrm{p}] @ 3 \mathrm{MeV}$ & $\mathrm{Y}_{n}[\mathrm{n} / \mathrm{p}] @ 5 \mathrm{MeV}$ & $\mathrm{T}_{f}\left[{ }^{\circ} \mathrm{C}\right]$ & $\sigma_{t h}[\mathrm{~W} / \mathrm{m} / \mathrm{K}]$ \\
\hline${ }^{7} \mathrm{Li}$ & 0.534 & 1.88 & $2.38 \times 10^{-4}$ & $1.01 \times 10^{-3}$ & 180 & 85 \\
${ }^{9} \mathrm{Be}$ & 1.848 & 1.85 & $5.23 \times 10^{-5}$ & $4.95 \times 10^{-4}$ & 1280 & 200 \\
\hline
\end{tabular}

is a significant gain. In addition, to maximize $\mathrm{Y}_{n}$, the target should have a high density $(\rho)$ and a high purity since impurities will only lead to proton energy losses and so, to a lower reaction rate. To have a thermal neutron flux $\left(\phi_{t h}\right)$ which can compete with a reactor flux, equal to $10^{6}-10^{8} \mathrm{n} / \mathrm{cm}^{2} / \mathrm{s}$ at the detector position, the proton beam intensity has to be around 20-100 mA. For $\mathrm{E}_{p}=3 \mathrm{MeV}$, this leads to a deposited power inside the target between $60 \mathrm{~kW}$ and $300 \mathrm{~kW}$. Since the lithium fusion temperature $\left(\mathrm{T}_{f}\right)$ and thermal conductivity $\left(\sigma_{t h}\right)$ are very low (Table 1$)$, operating a solid lithium target is difficult. It will only be possible with a large target, not suited to maximize neutron flux, or with a rotating target, with complex mechanics. Recently a liquid lithium target (LiLiT) has been successfully developed at the SARAF facility [5] and could be the key to operate a lithium target with high intensity beams. In the SONATE project, a solid beryllium target sustaining $50 \mathrm{~kW}$ is studied in priority. It has as advantages good thermal properties although $\mathrm{Y}_{n}$ is four times less than with lithium (Table 1). To optimize the TMR configuration Monte-Carlo simulations are performed. Considering the low neutron yields, to reduce the computation time ${ }^{9} \mathrm{Be}(\mathrm{p}, \mathrm{n})$ reactions are not simulated. A neutron source term is built by hands based on experimental data (see Section 3). One drawback of using experimental data is that the emitted neutron energy and angle are not kinematically correlated. This adds to the simulations an uncertainty.

\section{Experimental results and Geant4 simulation validation}

To develop SONATE, different steps are being followed. In 2016, experiments where performed with a $10 \mathrm{~W}$ beam. These have firstly validated the experimental neutron angular distribution from Howard [6] and discarded Marion's one [7] as shown in [8]. The former is thus used as input in the Geant 4 simulation. The neutrons are preferentially emitted at 83 degrees w.r.t the beam axis. The neutron energy distribution is built from Howard data [6] and has an average energy of $640 \mathrm{keV}$. More details are given in [9]. With these ingredients, in the 2016 TMR configuration presented in Figure 1 (left), the simulation agrees with the experimental data with an accuracy better than $20 \%$ as shown in Figure 2 (IPHI-2016 legends). The Geant 4 simulation has also been successfully benchmarked against MCNP6 [10]. This validates the neutron transportation process in Geant4. In 2019, an experiment to test the thermal resistance and lifetime of a solid beryllium target with a $3 \mathrm{~kW}$ beam has been performed. The inherent difficulty in operating such a target with a high beam intensity (here $1 \mathrm{~mA}$ in average) is the proton blis- tering effect. A high number of protons is implanted in the target and since the hydrogen solubility in Be and its diffusion coefficient are low, hydrogen atoms start to accumulate. Blisters appear and damage the target. With the beryllium target used for the experiment no blistering effect has been observed so far. The main difference regarding the 2016 and 2019 TMR experimental configurations are sketched in Figure 1 and summarized in Table 2. The Geant 4 simulation also agrees with the data taken in the 2019 configuration inside their uncertainties as presented in Figure 2 (IPHI-2019 legends).

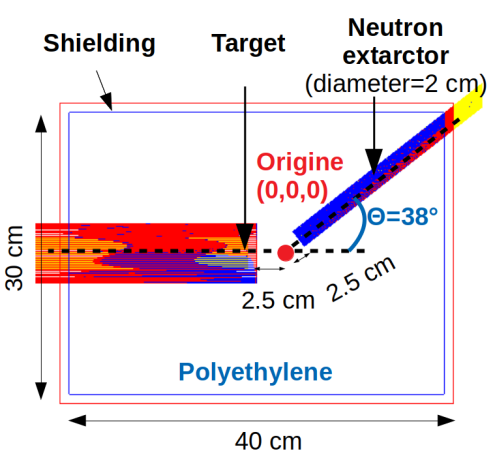

2016 TMR configuration

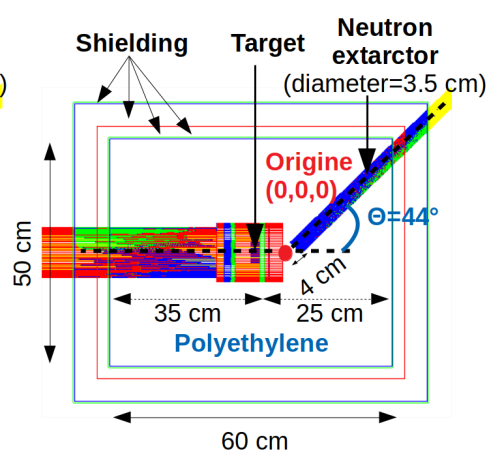

2019 TMR configuration
Figure 1: Sketches of the 2016 (left) and 2019 (right) TMR experimental configurations. The origin of the distance to neutron detectors is also indicated.

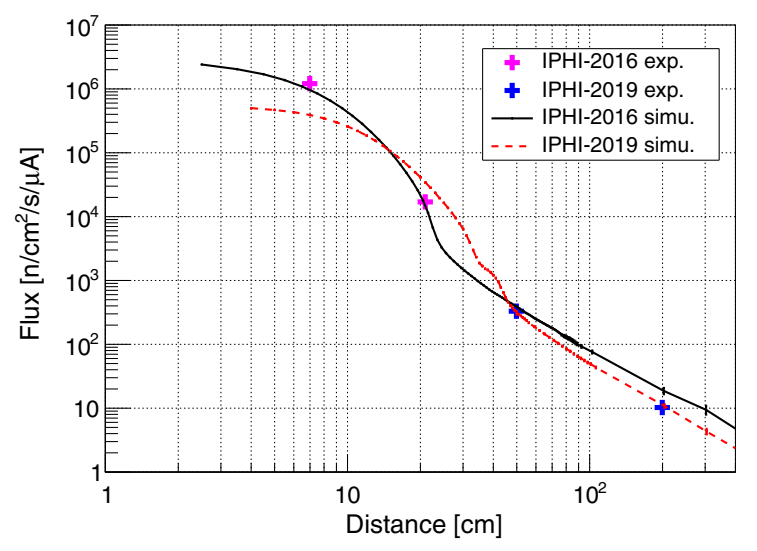

Figure 2: Thermal neutron flux $\left(E_{n}<400 \mathrm{meV}\right)$ as a function of the distance (see Figure 1), for the 2016 and 2019 TMR configurations. Geant4 simulation results are compared with experimental data. 
Table 2: Main differences between the 2016 and 2019 TMR experimental configurations performed at IPHI.

\begin{tabular}{c|ccc|cc|c|c}
\hline & \multicolumn{3}{|c}{ Accelerator } & \multicolumn{2}{c}{ Target } & $\begin{array}{c}\text { Polyethylene } \\
\text { moderator }\end{array}$ & Extractor \\
\hline & Power & $\begin{array}{c}\text { Tube } \\
\text { diameter }\end{array}$ & $\begin{array}{c}\text { Beam } \\
\text { diameter }\end{array}$ & Material & Backing & Dimensions & Axial distance \\
\hline 2016 & $10 \mathrm{~W}$ & $60 \mathrm{~mm}$ & $16 \mathrm{~mm}$ & $0.5 \mathrm{~mm}$ & $\mathrm{Al}: 10 \mathrm{~mm}$ & $30 \times 30 \times 40 \mathrm{~cm}^{3}$ & $2.5 \mathrm{~cm}$ \\
\hline 2019 & $3.0 \mathrm{~kW}$ & $110 \mathrm{~mm}$ & $50 \mathrm{~mm}$ & $20 \mathrm{~mm}$ & $\begin{array}{c}\mathrm{Cu}: 10 \mathrm{~mm} \\
\mathrm{Al}: 10 \mathrm{~mm}\end{array}$ & $50 \times 50 \times 60 \mathrm{~cm}^{3}$ & $4 \mathrm{~cm}$ \\
\hline
\end{tabular}

Between the 2016 and 2019 TMR configurations (Figure 2), at 2 meters, the simulated $\phi_{t h}$ dropped by a factor 1.7. This is mainly because the target thickness increases from $0.5 \mathrm{~mm}$ to $20 \mathrm{~mm}$ (Table 2). Indeed, in beryllium, a $640 \mathrm{keV}$ (average initial neutron energy) neutron has a mean free path around $17 \mathrm{~mm}$ and the neutron mean free path averaged over the initial neutron energy spectrum is around $22 \mathrm{~mm}$. These are similar to the $20 \mathrm{~mm}$ target thickness. Therefore, in average neutrons interact once before reaching the moderator. This spatially dilutes the neutrons leading to a less efficient neutron extraction. This target thickness was initially chosen for mechanical and thermal constraint reasons. This underlines the need to find the best compromise between mechanical, thermal, and neutronic constraints to maximize the neutron flux.

\section{Moderator and reflector design}

To provide the highest well collimated thermal flux outside the TMR assembly, the moderator and reflector materials and geometries, along with their coupling, have to be carefully investigated. During this optimization process, it has been systematically checked that the moderator/reflector material descriptions take into account the thermal scattering law (TSL) necessary to accurately transport thermal neutrons. In Geant4 (version 10.04.p02), TSL are taken from the ENDF/B-VII.0 data library [11].

Good moderator and reflector materials should both have a low capture cross-section and a high elastic crosssection. In addition, the moderator material should have a low nuclear mass to maximize the energy transfer between the neutron and the recoil nucleus. This is to minimize the number of collisions to thermalize the neutrons and so to minimize the neutron spatial dilution. Regarding the cross-sections in JEFF-3.3 data librairy, the best moderator materials are found to be polyethylene, light water, heavy water and graphite. These were expected based on previous reactor physic studies as summarized in $[12,13]$. In the following, the TMR configuration is the 2016 one with the 2019 accelerator/target part (Figure 1). Starting from this configuration only the moderator material is changed. As expected, Figure 3 shows that for a given moderator volume and neutron extractor geometry, more thermal neutrons are extracted with hydrogenous materials. For heavy water and graphite larger dimensions are needed to thermalize the neutrons. This dilutes the spatial neutron distributions, which is not ideal to maximize $\phi_{t h}$ for collimated beam when only one extraction chan- nel is used. The thermal fluxes obtained with polyethylene $\left(\mathrm{CH}_{2}\right)$ and light water $\left(\mathrm{H}_{2} \mathrm{O}\right)$ are similar (Figure 3). However the thermal beam quality, defined by $\phi_{t h} / \phi_{t o t}$, is higher by a factor 1.5 with $\mathrm{CH}_{2}$ compare to $\mathrm{H}_{2} \mathrm{O}$ because ${ }^{16} \mathrm{O}$ has a higher mass than ${ }^{12} \mathrm{C}$. To conclude, polyethylene is the best moderator to get a well collimated beam along with a high thermal neutron flux for one extraction channel and a low beam power. For higher beam power $\mathrm{H}_{2} \mathrm{O}$ will be considered because of the $\mathrm{CH}_{2}$ low thermal conductivity. For multiple beam extractions, $\mathrm{D}_{2} \mathrm{O}$ could be also a good candidate.

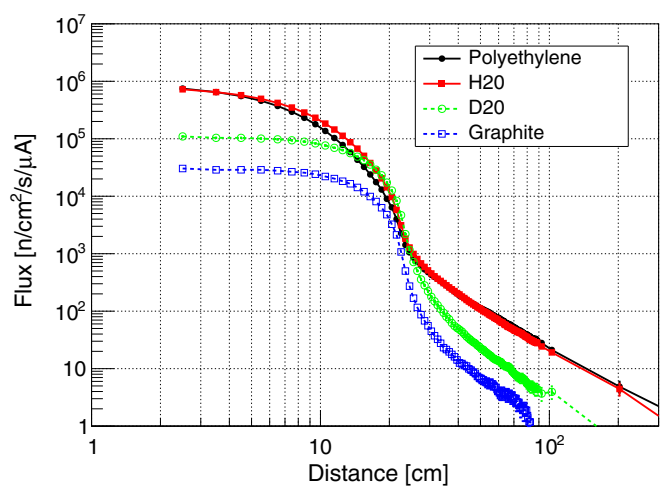

Figure 3: Thermal neutron flux as a function of the distance (see Figure 1), for different moderator materials. The TMR configuration is the 2016 one with the 2019 accelerator/target part.

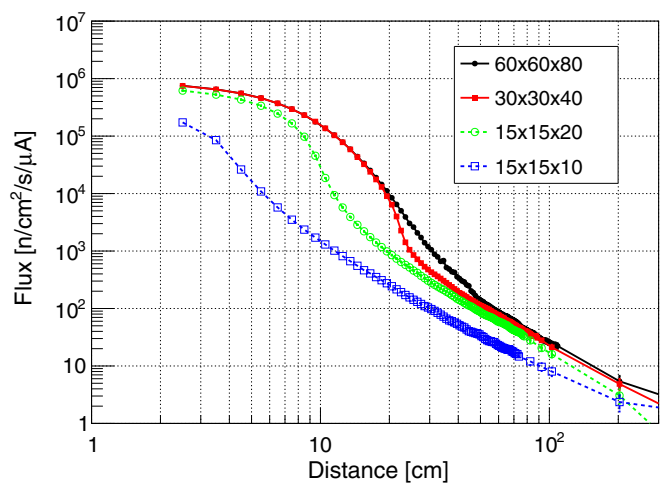

Figure 4: Thermal neutron flux as a function of the distance (see Figure 1), for different polyethylene moderator dimensions. 
The impact of the polyethylene moderator dimensions on $\phi_{t h}$ has then been studied. Figure 4 shows that the bigger the moderator size is, the higher $\phi_{t h}$ is (neutrons are more thermalized). It also shows that starting from a $30 \times 30 \times 40 \mathrm{~cm}^{3}$ moderator, $\phi_{t h}$ starts to converge to an asymptotic value as the moderator size increases. It is then not necessary to increase the moderator volume above this volume.

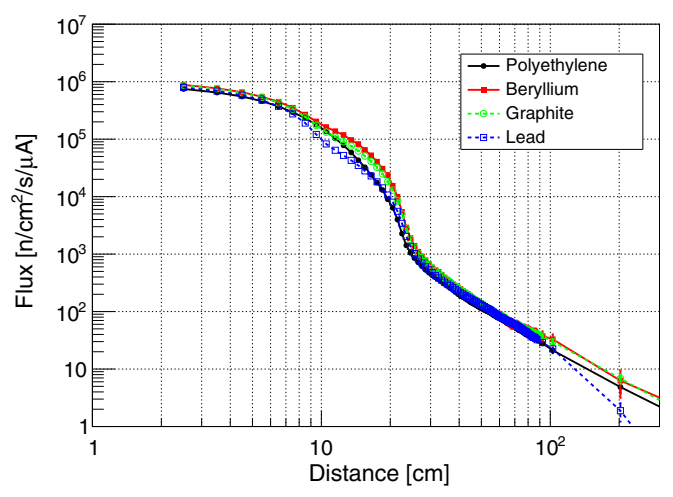

Figure 5: Thermal neutron flux as a function of the distance, for different reflector material. In this configuration $a \sim 10 \mathrm{~cm}$ thick reflector material is placed around a $15 \times 15 \times 20 \mathrm{~cm}^{3}$ polyethylene moderator.

To increase the thermal neutron flux outside the TMR assembly, a reflector can be placed around the moderator. Its role is to send back the neutrons to the moderator volume. The quality of a reflector is given by its albedo parameter $(\beta)$ (details are given in $[12,13]$ ). For a reflector size around three times the neutron diffusion length, $\beta$ converges toward an asymptotic value:

$$
\beta_{a s}=\frac{1-2 \sqrt{\Sigma_{a} / 3 \Sigma_{t r}}}{1+2 \sqrt{\Sigma_{a} / 3 \Sigma_{t r}}}
$$

with $\Sigma_{t r}=\Sigma_{t o t}-\bar{\mu} \Sigma_{s}, \Sigma_{t o t}, \Sigma_{s}$ and $\Sigma_{a}$ the transport, total, scattering and absorption macroscopic cross-sections. $\bar{\mu}=2 / 3 A$ is the average cosine of the scattering neutron angle in the laboratory frame. $A$ is the nuclear mass. From Equation (1), a good reflector is characterized by a low $\Sigma_{a}$ and a high $\Sigma_{s}$. Heavy water, beryllium, graphite and lead (which is also a gamma shielding) can be used as a reflector. Heavy water is not considered anymore because its thermal neutron diffusion length is more than 1 meter, which will result in having a very large reflector. Taking into account these considerations, a $15 \times 15 \times 20 \mathrm{~cm}^{3}$ polyethylene moderator is wrapped in a $\sim 10 \mathrm{~cm}$ thick reflector. Figure 5 presents the impact of the reflector material on the thermal flux. At 1 meter, $\phi_{t h}$ is higher by a factor 1.5 with beryllium and graphite compare with polyethylene and lead. Polyethylene is a poor reflector because of its high capture cross-section. Knowing that $\beta_{a s}$ is reached for a material thickness equal to three times the thermal neutron diffusion length in the material, calculations showed that for beryllium and graphite, $\beta_{a s}$ are respectively reached for a $60 \mathrm{~cm}$ and $165 \mathrm{~cm}$ reflector thickness. In increasing the beryllium reflector thickness from $10 \mathrm{~cm}$ to $60 \mathrm{~cm} \phi_{t h}$ is expected to increase approximately by a factor 2 . This has to be confirmed by experiments.

\section{Conclusion}

Since the Orphée reactor will shutdown in 2019, an alternative solution to provide neutron beams is being investigated in CEA-Saclay. Taking advantage of the IPHI high intensity proton accelerator, a compact accelerator driven neutron source is expected to be developed. The first experiments performed from 2016 to 2019 allowed to gain experience in operating solid beryllium targets with high intensity proton beams and to validate the Geant4 simulation software for two TMR configurations. The first optimization steps have shown that a TMR configuration with a small polyethylene moderator coupled to a big beryllium (or graphite) reflector will maximize the thermal neutron flux outside the TMR assembly for one neutron extractor. In 2020, the average beam intensity will be increased from $1 \mathrm{~mA}$ to $17 \mathrm{~mA}$. The deposited power in the target will be around $50 \mathrm{~kW}$. As the power density limit is set to $0.5 \mathrm{~kW} / \mathrm{cm}^{2}$, the target has to be redesigned. New studies are ongoing to find the best TMR configuration for this new target. In a near future, cold moderator and neutron extractor geometries will be investigated to increase the neutron flux and to develop new TMR configurations dedicated to other neutronic instruments. In a longer future, the accelerator will be upgraded to provide $20 \mathrm{MeV}$ protons.

\section{References}

[1] E.P. Sciences, E.S.W.G.N.L. Group, p. 75 (2016)

[2] J. Allison et al., Nucl. Instr. Meth. Phys. Res. A 835, 186 (2019)

[3] K. Porgès et al., APPLIED PHYSICS DIVISION ANNUAL REPORT ANL-7910, 361 (1970)

[4] H. Gibbons, J. R. L. Macklin, Phys. Rev. 114, 571 (1959)

[5] M. Paul et al., Eur. Phys. J. A 55, 44 (2019)

[6] W. Howard, J. Yanch, Nucl. Sci. pp. 145-160 (2001)

[7] J.B. Marion, Phys. Rev. 103, 713 (1956)

[8] H. Tran et al., J. Phys. Conf. Ser. 1021, 012008 (2018)

[9] A. Letourneau et al., EPJ Web Conf. 146, 03018 (2017)

[10] D. B. Pelowitz et al., MCNP6TM User's manual Version 1.0 (2013)

[11] M. B. Chadwick et al., Nuclear Data Sheets 107, 2931 (2006)

[12] J. R. Lamarsh, Introduction to Nuclear Reactor Theory (ADDISON-WESELEY PUBLISHING COMPANY, 2001)

[13] P. Reuss, Précis de neutronique (EDP Sciences, 2003) 